\title{
Die Furcht vor der Freiheit, kritisch zu denken
}

\section{Einführung}

Richter sind unabhängig und nur dem Gesetz unterworfen. Auch einer Dienstaufsicht unterstehen sie nur insoweit, als dadurch ihre Unabhängigkeit nicht beeinträchtigt ist. Diese wunderbare Freiheit postuliert das deutsche Richtergesetz, gestützt auf die rechtsstaatliche Ordnung des Grundgesetzes, die ihrerseits in der langen Traditionslinie aufgeklärten Denkens steht.

Wie viel diese Freiheit in der Praxis wert ist, wenn eine junge Assessorin oder ein junger Assessor als Beisitzer in einer Landgerichtskammer unter den strengen Blicken des Vorsitzenden das „dritte Juristische Staatsexamen“ zu bestehen hat, steht auf einem ganz anderen Blatt. Als Trost bleibt, dass später, nachdem die junge juristische Karriere die Probe bestanden hat, noch viel Raum und Zeit für die versprochene Freiheit bleibt. Und ist es denn wirklich so schlimm, wenn man sich einstweilen in Opportunismus übt? Einfach das nachbetet, was die einen vorformuliert haben und die anderen von einem hören möchten? Doch sicher nicht. Schon viele Juristengenerationen zuvor hatten diese Subordination zu leisten, ohne dass dadurch die Rechtsordnung einen nennenswerten Schaden erlitten hätte.

Was wäre aber, wenn man nie gelernt hätte, diese Freiheit mit lebendigen Inhalten zu füllen? Wo und wie lässt sich eigentlich der Gebrauch dieser Freiheit erlernen? Die Frage nach dem ,Wo' ist schnell und einfach zu beantworten. Natürlich an der Universität. Denn wo sonst ist es möglich, seine vielleicht noch etwas ungeübte kritische Fantasie walten zu lassen, ohne dadurch gleich einem Menschen oder einem Wirtschaftsunternehmen finanzielle Schäden zuzufügen. Schwieriger zu beantworten ist allerdings die Frage nach dem, Wie'. Vielleicht aber nur deshalb, weil es hierauf keine einheitliche Antwort gibt. Viele unterschiedliche Wege können zu dem Ziel führen, sich für den sachgerechten Gebrauch der juristischen Freiheit zu bilden. Es ist in diesem Sinne durchaus willkürlich, dass nachfolgend die Rechtsgeschichte als Gegenstand und Beispiel für die Schulung eines kritischen Verstandes ausgewählt wurde. Im selben Sinne befruchtend wirken können ebenso die Philosophie, die Soziologie, die Rechtsvergleichung oder die Ökonomie. Vielleicht hebt sich die Rechtsgeschichte aber doch ein wenig aus dem Kanon der Grundlagenfächer hervor. So bedarf es nur eines Blickes um wenige Jahrzehnte zurück, um festzustellen, dass Opportunismus und der kritiklose Umgang mit Autoritäten eben doch Verhaltensweisen sind, durch die einer Rechtsordnung schwerer Schaden zugefügt werden kann. Entsetzt blickte Gustav Radbruch nach dem Ende des Zweiten Weltkriegs auf die schrecklichen Folgen des Umstandes zurück, dass „der Positivismus“ die deutschen Juristen so offenbar blind und ,wehrlos“ gegenüber den Gräueln der nationalsozialistischen Herrschaft gemacht hatte. ${ }^{1}$

1 Vertiefend zum Kontext der berühmten "Radbruch-These": Walther, Manfred, Hat der juristische Positivismus die deutschen Juristen im "Dritten Reich" wehrlos gemacht?, in: Dreier/Sellert (Hrsg.), Recht und Justiz im "Dritten Reich", S. 349 f. 


\section{Anlass zur Kritik}

Ein Blick auf die deutsche Privatrechtslandschaft der Gegenwart bietet reichlich Anlass für Kritik. An erster Stelle zu beklagen ist wohl der Verlust an interessenorientierter Systematik in Gesetzgebung, Rechtsprechung und vor allem im Rahmen studentischen Lernens. Bereits die als auflockernde Einstiegsfrage im Juristischen Staatsexamen konzipierte Frage, welches denn die Hauptregelungsgegenstände des Allgemeinen Teils des BGB seien, macht manchen Kandidaten nervös oder stürzt ihn gar in Verzweiflung. Regelmäßig wird als Antwort die Verjährung genannt und erst auf die Nachfrage, ob die Verjährung denn so bedeutend sei, dass sie an erster Stelle genannt zu werden verdient, kommt dann manchmal ein Denkprozess mit dem Ergebnis in Gang, dass Personen, Sachen und Rechtsgeschäfte eben doch Rechtsinstitute von weit zentralerer Bedeutung sind. Ähnliche Beobachtungen lassen sich bei den Fragen machen, was denn ein Schuldverhältnis sei oder ein Rechtsgeschäft und worin jeweils die Gemeinsamkeiten und die Unterschiede zu einem Vertrag bestehen könnten.

Das kleine Beispiel deckt die Defizite auf, die heute in weiten Bereichen das juristische Lernen und Arbeiten kennzeichnen: Der Aneignung von Systematik wird recht wenig Aufmerksamkeit geschenkt, weil ihr Wert nicht erkannt wird. An Stelle eigenständigen Lernens durch systematische Ermittlung und Verarbeitung des relevanten Stoffes ist viel zu oft eine schlichte Einlagerung vorgefertigten Ergebniswissens zu beobachten. Alles scheint sich allein darauf zu konzentrieren, sich einzuprägen, wie der BGH eine bestimmte Frage entschieden hat. Das Gefühl, dass es im Examen allein darauf ankomme, vermitteln leider viele Repetitoren und bestätigen leider einige Prädikatsabsolventen.

Dass auch die Produkte höchstrichterlicher Rechtsprechung durchaus eine kritische Betrachtung verdienen, zeigt sich an folgendem Beispiel. Angestoßen durch eine Entscheidung des BGH hat sich die deutsche Privatrechtswissenschaft in jüngerer Zeit verstärkt mit der Frage beschäftigt, welcher Rechtsnatur das Flaschenpfand bei Mehrweggetränkeflaschen sei. ${ }^{2}$ Konkret, ob ein Vertreiber von „Pfandflaschen“ auch nach der Veräußerung deren Eigentümer bleibt. Die Sachverhaltskonstellation, insbesondere die wirtschaftlichen Interessen der Beteiligten sprechen generell eher für eine Veräußerung mit unbeschränktem Eigentumsübergang auf den Erwerber, verknüpft mit einem Rückerwerbsversprechen des Veräußerers. Nach einer jüngst vom BGH vertretenen Ansicht $^{3}$ soll dagegen bei individualisierten Mehrwegflaschen das Eigentum tatsächlich beim Veräußerer verbleiben, sofern sie mit einer dauerhaften Kennzeichnung des Herstellers versehen sind. Nach Auffassung des BGH könne dieser sowohl Herausgabe seiner Flaschen, Schadensersatz für ihren Verlust und Unterlassung der Einbehaltung und Vernichtung durch Dritte beanspruchen.

Im Verhältnis zu einem möglicherweise rechtsmissbräuchlich handelnden Konkurrenzunternehmen vermag diese Ansicht noch eine gewisse Überzeugungskraft zu entfalten, doch ist der BGH mit seiner Entscheidung deutlich über dieses Ziel hinausgeschossen, indem er bestimmte: „Der Eigentümer einer individualisierten - auf Grund einer dauerhaften Kennzeichnung als sein Eigentum ausgewiesenen - Mehrwegflasche

2 Weber, Jörg-Andreas, Die Rechtsnatur des Flaschenpfands, NJW 2008, S. 948-953 m. w. N.

3 NJW 2007, S. 2913-2916 = JuS 2007, S. 1060-1062. 
verliert das Eigentum an der Flasche weder durch den Verkauf des Getränks an den Großhandel noch durch den weiteren Vertrieb des Getränks bis zum Endverbraucher." ${ }^{4}$ Es ist kaum vorstellbar, welche Folgen es hätte, wenn sich diese Auffassung tatsächlich im Rahmen der deutschen Rechtsordnung durchsetzen würde. Die Staatsanwaltschaften hätten dann zum Beispiel Bürger, die eine Getränkeflasche mutwillig zerstören wegen Sachbeschädigung zu verfolgen oder diejenigen, die eine solche Flasche auf Dauer behalten würden, beispielsweise um diese als dekorativen Kerzenständer $\mathrm{zu}$ verwenden, wegen der dadurch begangenen Unterschlagung zu belangen.

Vielleicht gerade wegen der alltäglichen Bekanntheit der Sachverhaltskonstellation ist die Pfandflaschenproblematik zu einem recht beliebten Thema für juristische Hausarbeiten geworden. ${ }^{5}$ Bei der Betrachtung der Hausarbeiten fällt auf, dass nur außerordentlich wenige Bearbeiter den Mut finden, den Ergebnissen des BGH nicht zu folgen, ja diese auch nur ernsthaft kritisch zu hinterfragen. Besondere Bedeutung kommt dabei einer in der höchstrichterlichen Entscheidung verwendeten Begründung zu, die nur scheinbar Überzeugungskraft enthält: Jeder Besitzer der Mehrwegflaschen könne und müsse erkennen, dass diese nicht sein Eigentum, sondern Eigentum des Herstellers seien, da ,gerade aus der individuellen Kennzeichnung der Flaschen der Wille des Herstellers erkennbar" sei, ,die Flaschen zurückzubekommen und sie deshalb nur zur vorübergehenden Benutzung und nicht zu Eigentum zu überlassen. "6 Völlig verkannt wird, dass es sich hierbei lediglich um ein Scheinargument handelt, das auch durch seine häufige Wiederholung nicht an Überzeugungskraft gewinnt. Die dauerhafte Kennzeichnung von Produkten mit Herstellernamen oder -zeichen ist eine weit verbreitete Folge der Beliebtheit von Markenprodukten jeglicher Art. Ein Produkt wird in unserer Gesellschaft häufig einem vergleichbaren Gegenstand vorgezogen, gerade weil ein bestimmter beliebter Hersteller dieses mit seinem Namen oder Zeichen versehen hat. Niemand käme ernsthaft auf den Gedanken, aus dieser Produktkennzeichnung auf ein fortbestehendes Eigentumsrecht des Herstellers zu schließen, beispielsweise bei einem Kraftfahrzeug, einem Fernsehgerät oder einem Kleidungsstück. Zu hinterfragen wäre, warum die höchstrichterliche Rechtsprechung ausgerechnet bei den Mehrwegflaschen eine Ausnahme von dieser Regel machen will. ${ }^{7}$

Das in dem Beispiel erkennbare, unreflektierte Festklammern an Einzelfallentscheidungen geht mit einer ebensolchen Einstellung gegenüber gesetzlichen Normierungen einher. Hilflosigkeit besteht bei allen Fragen, die nicht ausdrücklich im Gesetz beantwortet sind. Verstärkt seit Einführung der Schuldrechtsreform ist ein abnehmendes Verständnis erkennbar, dass eine Rechtsfrage oder gar eine Anspruchsgrundlage möglicherweise nicht im Gesetz ausformuliert ist. Selbst im Examinatorium, am Ende des

4 BGH NJW 2007, S. 2913.

5 An der Frankfurter Goethe-Universität war die Thematik in Wintersemestern 2007/2008 und 2009/2010 Gegenstand von Fortgeschrittenenhausarbeiten zum Sachenrecht.

6 BGH NJW 2007, S. 2914.

7 Auch bei anderen dogmatischen Fragen ist in jüngerer Zeit im universitären Unterricht zu beobachten, dass reines Ergebniswissen und unkritische Autoritätsgläubigkeit zu einer tiefen juristischen Hilflosigkeit führen können, wenn zu einem bestimmten Rechtsproblem keine höchstrichterliche Rechtsprechung oder herrschende Meinung ermittelbar ist. Die Notwendigkeit in solchen Fällen selbständig auf der Grundlage der erlernten juristischen Systematik eine Entscheidung treffen zu müssen, wird dann schnell als Überforderung bewertet. 
Studiums sind nicht jedem Hörer die Unterschiede zwischen „Recht“ und „Gesetz“ präsent, vielleicht sogar noch weniger, als den Erstsemestern.

Auch in wissenschaftlichen Arbeiten ist inzwischen vereinzelt die Überzeugung anzutreffen, dass rechtliche Gegebenheiten erst dann zufriedenstellend erfasst sind, wenn sie gesetzlich erfasst sind. Für die Bereiche des Strafrechts und des öffentlichen Rechts mag eine solche Auffassung eine gewisse Berechtigung haben, für das Zivilrecht ist sie jedoch nicht sehr zielführend.

Als Beispiel sei eine Untersuchung mit dem Titel „Versandhandel und Verbraucherschutz - Entstehung und Genese in rechtshistorischer Perspektive" aus dem Jahr 2005 genannt. ${ }^{8}$ Bereits im ersten Drittel der Arbeit hat der Verfasser eigentlich alle wesentlichen Ergebnisse ermittelt: Schon im ausgehenden 19. Jahrhundert hätten die Versandunternehmer ihren Kunden auf freiwilliger Basis ein Widerrufsrecht eingeräumt. Auch weitere versandhandelstypische Probleme, wie die Zusendung unbestellter Ware und die jeweiligen Gefahrtragungsregeln seien schon in dieser frühen Zeit erkannt und sachgerecht durch ein hohes Maß an Kulanz gelöst worden. ${ }^{9}$ Trotz dieses aussagekräftigen und vielleicht auch überraschenden Ergebnisses wendet sich der Verfasser nachfolgend auf fast zweihundert Seiten der Frage zu, warum eine spezialgesetzliche Regelung des Versandhandelsrechts ,erst so spät“, Ausgangs des 20. Jahrhunderts erfolgt ist. Er offenbart damit ein nicht unproblematisches zivilistisches Vorverständnis über das Verhältnis und die Aufgabenverteilung zwischen Recht und Gesetz. Der Verfasser führt aus, dass die so späte, erst am Ausgang des zwanzigsten Jahrhunderts erfolgte, spezialgesetzliche Regelung des Fernabsatzrechts ,,jedenfalls nicht“ dadurch zu erklären sei, dass der Versandhandel etwa erst in dieser Zeit als neues Phänomen in Erscheinung getreten sei. Vielmehr sei erst ein „erheblicher Problemdruck“ Grundvoraussetzung für die Schaffung von „konsumentenschützenden Rechtsnormen“ gewesen. Im Versandhandel habe dieser lange ,gefehlt", weil er zum einen erst spät ein ,gesamtgesellschaftliches Phänomen“ geworden sei und zum anderen die frühen Versandhändler „,ein hohes $\mathrm{Maß}$ an Kulanz" gezeigt hätten. ${ }^{10}$

Eigentlich ist das wesentliche Untersuchungsergebnis mit diesen Feststellungen bereits gefunden. Es stellt sich die Frage, warum der Verfasser in den Folgekapiteln so starr und unnachgiebig auf die spezialgesetzliche Normenbildung fixiert bleibt. Wenn doch die tatsächlichen Verhältnisse und das jeweils geltende Recht, ob gewohnheitlichen oder allgemeinrechtlich fixierten Ursprungs, so offensichtlich und problemlos alle juristischen Möglichkeiten bereithält, die in der Praxis auftretenden Konfliktfälle adäquat zu lösen, welcher Sinn und Zweck sollte denn dann überhaupt noch einer entsprechenden Spezialnorm zukommen?

8 Stolte, Stefan, Versandhandel und Verbraucherschutz. Entstehung und Genese in rechtshistorischer Perspektive (Forschungen zur Neueren Privatrechtsgeschichte Bd. 30), Köln u. a. 2005. Rezension der Arbeit in: Forum historiae iuris, erste europäische Internetzeitschrift für Rechtsgeschichte, http://www.forhistur.de/zitat/ 0605frassek.htm).

9 Ein freiwilliges Widerrufsrecht sei bereits um 1900 gewährt worden (S. 43), mit dem Inkrafttreten des BGB hätten Versandkäufe im Rechtsinstitut des Kaufs auf Probe ( $\S 459$ f. a. F.) ihre dogmatische Heimat gefunden (S. 63-70) und auch versandhandelstypische Spezialprobleme der Zusendung unbestellter Ware (S. 71-81), der Gefahrtragung (S. 81-88) und der Gebrauch allgemeiner Geschäftsbedingungen (S. 88-97), Stolte, Versandhandel.

10 Stolte, Versandhandel, S. 100. 
Die Frage, warum ,ein entsprechendes Regelungswerk nicht bereits früher entstanden ist", ${ }^{11}$ erscheint danach müßig, denn die in der Untersuchung erzielbaren Antworten liegen auf der Hand: die Schaffung ,eines einheitlichen, Verbraucher schützenden Versandhandelsrechts“ sei „deshalb nicht vorgenommen“"worden, ,weil faktisch bereits ein hohes Niveau an Verbraucherrechten herrschte. “12 „Eine spürbare Steigerung des Verbraucherschutzniveaus“ konnte ,aus der Perspektive des deutschen Gesetzgebers nicht konstatiert werden.“13 „Aus der Perspektive eines situativen Verbraucherschutzmodells könne man daher sagen, dass das Fernabsatzrecht bereits jetzt obsolet ist." 14 Trotz der sowohl rechtshistorischen wie europarechtlich intendierten Ausrichtung der Untersuchung, verharrt sie bei der Frage nach Spezialnormen deutlich in den engen Bahnen, die leider in der zweiten Hälfte des zwanzigsten Jahrhunderts charakteristisch für das deutsche Recht geworden sind. Konkret bei der Auffassung, dass ein juristisches Problem erst und nur dann adäquat gelöst sei, wenn der Gesetzgeber eine ausdrückliche Regelung formuliert habe. Dieser Auffassung ist entgegenzuhalten, dass ein Rechtsproblem bereits dann als gelöst anzusehen ist, wenn es der Rechtsprechung möglich ist, auf der Grundlage der geltenden Gesetze und des geltenden Rechts, die konkret in der Praxis auftretenden Konfliktfälle einem adäquaten Interessenausgleich zuführen zu können. Einer ausformulierten Norm bedarf es hierfür gerade nicht. Dies zeigt sowohl ein geschichtlicher Rückblick auf die deutschen Rechtsverhältnisse in der Zeit vor 1900 wie ein aktueller Blick auf den angloamerikanischen Rechtskreis.

Die Rechtsfindung in einem solchermaßen weitgehend „normenleeren Raum“ stellt allerdings hohe Anforderungen an die Juristen. Erforderlich ist ein umfassendes und tiefgreifendes Verständnis für die jeweiligen Interessen der Konfliktbeteiligten einerseits und für die systematischen Verbindungen der Rechtsinstitute andererseits. Gutes Recht erfordert verständnisvolle, verantwortungsvolle und umfassend gebildete Juristen. Dabei handelt es sich um juristische Tugenden, die auch in den kodifikationsorientierten kontinentaleuropäischen Rechtsordnungen nicht vernachlässigt werden sollten.

Gerade in Bezug auf die systematische Erfassung und Lösung von Rechtsproblemen kann einer wohlüberlegten Rechtsetzung eine Vorbildfunktion und damit auch eine große Verantwortung zukommen. Traditionell zeigt sich das System des deutschen Privatrechts vorrangig tatbestandsorientiert. Der exakten Erfassung und Abgrenzung von Sachverhaltsfragen durch den Normtext wurde stets sehr große Aufmerksamkeit geschenkt. War diese Arbeit getan, die Norm wohlüberlegt formuliert und der Sachverhalt sorgfältig unter ihren Tatbestand subsumiert, zeigte sich der Eintritt der Rechtsfolge fast automatisch sowohl folgerichtig als auch sachgerecht. Viele Jahrhunderte lang immer wieder erprobte Denkmodelle bildeten die feste Grundlage dieses juristischen „Gebäudes“. Typisierung und Abstraktion waren die Werkzeuge, die das Gebäude schlank und überschaubar hielten.

In der Gegenwart bietet das privatrechtliche Gebäude weder einen besonders schlanken Anblick, noch ist es besonders überschaubar und leider auch immer weniger tatbe-

11 Stolte, Versandhandel, S. 8.

12 Stolte, Versandhandel, S. 208 f., hier für den Zeitraum zwischen 1949 und 2000.

13 Stolte, Versandhandel, S. 237, für die Zeit kurz vor Umsetzung der Fernabsatzrichtlinie.

14 Stolte, Versandhandel, S. 245, Schlusssatz der Untersuchung. 
standlich systematisch durchdacht. Immer mehr scheint das Ergebnis, das Eintreten einer bestimmten gewollten Rechtsfolge, die tatbestandliche Stringenz und Systematik zu verdrängen. Teilweise orientiert sich die Rechtsetzung bei der Normgestaltung auch nicht mehr an den besonders typischen Interessenkonstellationen, sondern greift im Gegenteil sehr spezielle Einzelprobleme auf. Zu beobachten ist dies bei den Vorschriften zum Sachdarlehensvertrag, der nach $\S 607$ BGB nun regelmäßig als entgeltlicher Vertrag ausgestaltet ist. Der Grund dafür bestand darin, dass nicht mehr das ,ausleihen“ von Backzutaten an der Wohnungstür oder eines Sackes Zement für die Nachbarbaustelle, sondern die unter Banken und anderen Geldinstituten übliche „Wertpapierleihe“ die Ausgestaltung der Vorschriften durch den Gesetzgeber prägte.

Bis zur Schuldrechtsreform hatten die $\S \S 607$ ff. BGB Sach- und Gelddarlehen gemeinsam geregelt, indem dort neben Geld auch ,andere vertretbare Sachen“ genannt wurden. Grundsätzlich waren also die Vorschriften auf beide Darlehensformen gleichermaßen anwendbar. Im Zuge der Schuldrechtsmodernisierung verlor man diese Jahrhunderte alten systematischen Gemeinsamkeiten jedoch aus dem Auge. Zuviel Verbraucher schützendes Recht aus Brüssel hatte das Gelddarlehen inzwischen überlagert, so dass das „kleine Bäumchen“ des Sachdarlehens in diesem ,großen Wald“ kaum mehr erkennbar war und auch nicht mehr recht in diesen „Wald“ hineinpassen wollte. Trotz allem gestand der Gesetzgeber dem Sachdarlehen auch nach der Reform noch ein kleines Refugium zu, allerdings nicht ohne dass zuvor diskutiert worden wäre, es ganz herauszustreichen. ${ }^{15}$ „Im Bürgerlichen Gesetzbuch sollten sich jedenfalls die Grundprinzipien des Sachdarlehensvertrages finden, damit die Rechte und Pflichten aus einem solchen Vertrag für den Bürger ersichtlich“ seien. ${ }^{16}$ Diese Begründung vermag nur auf den ersten Blick zu überzeugen und erklärt nicht die nach der Reform als Regel angelegte Entgeltlichkeit des Sachdarlehensvertrages. Die wahren Gedanken ergeben sich dagegen aus der weiteren Gesetzesbegründung: Auf „die Regelungen des Sachdarlehens“ solle deshalb nicht verzichtet werden, weil es ,immerhin... in reiner Form bei der Wertpapierleihe zur Anwendung“" käme. ${ }^{17}$

Im Einklang mit der Begründung des Gesetzgebers belegt auch die Literatur, dass die Praxis der sog. „Wertpapierleihe“ die Neuregelung des Sachdarlehens im BGB erkennbar geprägt hat. ${ }^{18}$ Ein Wertpapierdarlehen, wie die korrekte Bezeichnung eigentlich lauten müsste, stellt eine der vielen Möglichkeiten dar, von kurzfristigen Kursschwankungen in der Börsenpraxis zu profitieren. Der Darlehensnehmer schafft sich die Möglichkeit, Wertpapiere an Kaufwillige zu übereignen, die er eigentlich nicht in seinem Bestand vorrätig hält. Anstatt diese Wertpapiere zuerst auf dem Markt zu erwerben und erst danach zu übereignen, werden die Papiere zunächst nur als Darlehen genommen, dann an den Käufer übereignet und erst zu einem viel späteren Zeitpunkt auf dem Markt erworben, um die Darlehensschuld zu begleichen. Der Darlehensnehmer spekuliert mit diesem Vorgehen auf einen mittelfristigen Kursverlust der Papiere, der es ihm später gestattet, die Papiere günstiger einzukaufen, als er sie an den Interessenten zuvor veräußert hatte. Der Darlehensnehmer steigert mit Hilfe der Wertpapierleihe seine Ertrags-

15 Deutscher Bundestag, 14. Wahlperiode, Drucksache 14/6040, S. 258.

16 Deutscher Bundestag, 14. Wahlperiode, Drucksache 14/6040, S. 259.

17 Deutscher Bundestag, 14. Wahlperiode, Drucksache 14/6040, S. 258.

18 Schäfer, Carsten, Das Sachdarlehen, in Haas, Lothar u. a., Das neue Schuldrecht, München 2002, S. 325. Palandt/Putzo, $\S 607$, Rn. 5. 
möglichkeiten bei fallenden Kursen. Der Vorteil des Darlehensgebers besteht darin, dass er, ohne überhaupt mit einem Käufer in Kontakt zu treten, über das Darlehensentgelt die Rendite seiner Wertpapiere steigern kann.

In erster Linie sind es Bankunternehmen, die sich gegenseitig Wertpapiere leihen. Gelegentlich auch im Ringtausch-Verfahren, mit dem Ziel, sich auf breitest möglicher Ebene gegen Kursverluste abzusichern. Im Einzelfall kann die spezielle Interessenlage auch dazu führen, dass Aktien ,geliehen“"werden, die nicht zum direkten Weiterverkauf benötigt oder im Bestand bereits vorgehalten werden. Wirtschaftliche Probleme entstehen in der Praxis jedoch weniger aus diesem Absicherungsverhalten der Banken, sondern aus der Vornahme echter „Leerverkäufe“ bei denen schließlich der eigene Vorteil aus der Spekulation auf fallende Börsenkurse gesucht wird. Dass durch eine solche Spekulation schwere Schäden für börsennotierte Wirtschaftsunternehmen hervorgerufen werden können, liegt auf der Hand. Auch für die Spekulanten kann das, was sich zunächst wie eine schlichte zeitliche Verschiebung von Erwerbs- und Verkaufsvorgang darstellt, zu schweren Konsequenzen führen. Denn durch diese Veränderung der Reihenfolge ist das Risiko nicht mehr wie sonst auf den Verlust des Anlagevermögens beschränkt, sondern theoretisch unbegrenzt. Zu ergänzen bleibt, dass die Wertpapierleihe und der Leerverkauf von Wertpapieren ein international verbreitetes Phänomen darstellen. Vor allem in den USA sind derartige Spekulationsgeschäfte, auch durch Privatanleger, üblich.

Dass derartige Bank- und Börsengeschäfte nur bei einer entgeltlichen Ausgestaltung des Sachdarlehensvertrages sinnvoll sind, ist offensichtlich. Für die Begründung, gerade diese Geschäfte als Grundlage und Regelfall für die Normgestaltung des BGB zu wählen, ist damit jedoch noch keine Aussage getroffen. Gerade die für die Bürger typischen und häufig auftretenden Sachverhaltskonstellationen, die sich durch ihre regelmäßige Unentgeltlichkeit auszeichnen, hat der Gesetzgeber vollkommen außer Betracht gelassen. Ebenso fern liegt die Vorstellung, dass den Bank- und Börsenunternehmen durch die spärlichen Sachdarlehensnormen des BGB neue rechtliche Erkenntnisse zu Teil würden. Wenig überzeugend ist auch die Begründung für die Beibehaltung der Terminologie. „Da sich der Begriff des Sachdarlehens im Sprachgebrauch eingebürgert“" habe, solle er ,,aufrecht erhalten bleiben. “19 Die Begründung überrascht, weil bekanntermaßen im allgemeinen Sprachgebrauch der „Bürger“ doch eher „leiht“" oder „,borgt“, und erst von den Juristen im Gespräch darüber belehrt wird, dass der „Leihwagen“ doch gemietet und die Schrauben oder Nägel des Heimwerker-Freundes eben nicht ,geliehen“, sondern allenfalls als Darlehen gegeben sind. Die Bezeichnung einer speziellen Sachdarlehensform als: „Wertpapierleihe“, selbst im nicht rechtsunkundigen Umfeld von Bank und Börse, spricht hier ebenfalls für sich selbst.

Die Reihe der Beispiele für nicht an regelorientierter Systematik und tatbestandlicher Schärfe, sondern allein am rechtsfolgenorientierten Einzelfallinteresse orientierter Normsetzung und Rechtsprechung lässt sich fortsetzen. So ist allein aus der Ergebnisund Rechtsfolgenperspektive zu erkennen, warum einem Rechtsanwalt über die culpa in contrahendo mit Schutzwirkung für Dritte (§ 311 III BGB) eine Haftung gegenüber Menschen auferlegt wird, die er nie zu Gesicht bekommen und mit denen er nie auch

19 Deutscher Bundestag, 14. Wahlperiode, Drucksache 14/6040, S. 259. 
nur ein einziges Wort gewechselt hat. ${ }^{20}$ Erst vor dem Hintergrund einer zahlungskräftigen Berufshaftpflichtversicherung werden die Gründe für eine solche Regelung und Einordnung deutlich, aber damit nicht unbedingt überzeugend. Teilweise gelangt die Rechtsprechung zu recht skurrilen Ergebnissen. Beispielsweise, indem einem Käufer trotz festgestellter Nichtigkeit des Vertrages noch zusätzlich ein verbraucherrechtliches Widerrufsrecht zugesprochen wird, um ihm die Vorteile dieses Rechtsbehelfs zukommen zu lassen. ${ }^{21}$ Oder wenn ohne inhaltliche oder sprachliche Bedenken der Kauf eines Schoßhundes oder eines Reitpferdes als „Verbrauchsgüterkauf“ qualifiziert wird. ${ }^{22}$

Bei der Betrachtung der jüngeren Normsetzung stellt sich nicht nur für die oben erörterte Sachdarlehensproblematik, sondern auch an anderen Punkten die Frage, welche Beweggründe die Überlegungen des Gesetzgebers wohl bei der Formulierung bestimmter Normen geleitet haben mögen. Besonders auffällig ist dies bei den durch die Schuldrechtsreform eingeführten Vorschriften zur Nacherfüllung im Kaufrecht, §§437 I Nr. 1 und 439 BGB. Die Formulierungen des Normtextes lassen die Nacherfüllung ganz eindeutig als ein Recht des Käufers auf Nacherfüllung erscheinen. Die Orientierung an den Inhalten der Regelung und den Interessen der Beteiligten führt jedoch mit derselben Eindeutigkeit genau zum gegenteiligen Ergebnis. Da der Verkäufer nach § 439 III BGB faktisch jederzeit die Nacherfüllung aus Kostengründen verweigern kann, wäre es im Sinne einer an den Parteiinteressen orientierten Systematik also angebracht, die Nacherfüllung nicht als Pflicht, sondern als ein Nacherfüllungsrecht des Verkäufers zu qualifizieren. Lediglich dem Verkäufer verschafft die Nacherfüllung aufgrund des Vorranges, der ihr gewährt wird, einen Vorteil, indem sie den anderen Käuferrechten auf Minderung, Schadensersatz oder Rücktritt vom Vertrag zwangsweise vorgelagert ist. Die Nichtbeachtung dieses Vorrangs entlastet den Verkäufer und schränkt den Käufer in seinen potentiellen Rechten ein. Der Käufer muss zunächst eine Nacherfüllung ermöglichen, bevor er weitergehende Rechte geltend machen kann. Die wahre Interessenlage wird durch die Formulierungen des Normtextes also eher verschleiert als beschrieben.

Die Reihe der angeführten Beispiele verdeutlicht, wie wichtig eine kritische Auseinandersetzung mit Norminhalten und der zu ihnen ergangenen Rechtsprechung ist. Dabei handelt es sich vorrangig um eine Aufgabe der rechtswissenschaftlichen Forschung und Literatur. Darüber hinaus ist es jedoch eine zentrale Aufgabe der Juristenausbildung, den Stimmen der Forschung auch entsprechendes Gehör zu verschaffen und die Bedeutung der diversen Mindermeinungen für eine sachgerechte Fortentwicklung des Rechts zu betonen. Der Begriff der Mindermeinung sollte nicht mit Minderwertigkeit der Meinung gleichgesetzt werden. Sowohl im Unterricht als auch bei der Bewertung von juristischen Hausarbeiten, die scheuklappenbehaftet auf das als einzig richtig empfundene Ergebnis der „herrschenden Meinung“ zusteuern, besteht Gelegenheit dafür. Gerade die Grundlagenwissenschaften Rechtsgeschichte, Rechtssoziologie und Rechts-

20 BGH, NJW-RR 2003, S. 1035. Zur Entscheidung: Frassek, Ralf, Umfang der Haftung eines vertraglichen Ratgebers und Einbeziehung Dritter in den Schutzbereich vorvertraglicher Pflichten - BGH, NJW-RR 2003, S. 1035, in: JuS 2004, S. 285-287.

21 BGH, NJW 2010, S. 610-612 mit Anmerkung von Mirko Möller. Zur selben Entscheidung: Faust, Florian, Widerruf eines sittenwidrigen Fernabsatzvertrages, JuS 2010, S. 442-444.

22 BGH, NJW 2006, S. 2250-2254 = JuS 2006, S. 930-933, mit Kommentierung von Volker Emmerich. 
philosophie sind geeignet, in der universitären Lehre Orientierungspunkte für ein tiefgreifendes, sachgerechtes und systematisches Rechtsverständnis zu vermitteln.

\section{Kritikfreude als juristische Tugend}

Als Beispiel für die Wichtigkeit einer kritischen Auseinandersetzung mit Autoritäten und Dogmen ist nachfolgend bewusst ein Beispiel gewählt worden, das nicht der jüngeren Vergangenheit angehört. Fast 500 Jahre zurückblickend soll das evangelische Eherecht des Reformationsjahrhunderts betrachtet werden. Die Eherichter des 16. Jahrhunderts standen vor der äußerst schwierigen Aufgabe, die individuellen Interessen junger Menschen, die miteinander die Ehe eingehen wollten, mit den damaligen gesellschaftlichen Erfordernissen und Ansprüchen einer christlichen Werteordnung in Einklang zu bringen. Gerade wegen ihres größeren zeitlichen Abstandes zur Gegenwart bietet diese Thematik überraschend viele und vielfältige höchst aktuelle Perspektiven und Anknüpfungspunkte zu gegenwärtig diskutierten Rechtsfragen.

Als Folge der Reformation wurde dem Eherecht durch Martin Luthers Ablehnung des kanonischen Rechts sowohl seine Normengrundlage als auch seine Gerichtsbarkeit, die zuvor die katholischen Bischöfe ausgeübt hatten, entzogen. Gerade aber das Eherecht zeigte sich am Übergang vom Mittelalter zur Neuzeit als ausgesprochen brisanter und konfliktträchtiger Rechtsbereich. Die gewandelten Bedürfnisse der Menschen, in erster Linie das verstärkte Streben nach individuellem und vor allem diesseitigem Glück, senkte spürbar die Bereitschaft, sich allzu strengen eherechtlichen Normvorgaben zu unterwerfen. Für den kursächsischen Staat, der zu den bedeutendsten im alten Reich zählte, galt es, mit möglichst effektiven Maßnahmen den unerwünschten Folgen dieses Strebens zu begegnen. ${ }^{23}$ Die gegenläufigen Zielsetzungen und Motivationen von Staatsführung und Untertanen ließen einer Vielzahl interessanter Streitbeilegungsstrategien und Konfliktlösungsmuster entstehen. ${ }^{24}$

Interessant ist, dass der mächtige frühneuzeitliche Territorialstaat seine durchaus vorhanden Möglichkeiten zur Normierung und Normdurchsetzung scheinbar nicht ausschöpfte. Es wurden zunächst keine materiell-rechtlichen Vorgaben formuliert und auch

23 Frassek, Ralf, Eherecht und Ehegerichtsbarkeit in der Reformationszeit - Der Aufbau neuer Rechtsstrukturen im sächsischen Raum unter besonderer Berücksichtigung der Wirkungsgeschichte des Wittenberger Konsistoriums, Tübingen 2005, S. $47 \mathrm{ff}$.

24 Die Aktualität dieses Themas zeigt sich in einem gegenwärtig angestrebten, interdisziplinären Verbundforschungsvorhaben zur gerichtlichen und außergerichtlichen Konfliktlösung, getragen von der Goethe-Universität, dem Max-Planck-Institut für europäische Rechtsgeschichte, der Forschungsstelle für Reichskammergerichtsforschung und der Fachhochschule Frankfurt. 
kein flächendeckendes Gerichtssystem aufgebaut. ${ }^{25}$ Ganz im Gegensatz zum kleinen fernen Zürich, in dem schon sehr bald nach Einführung der Reformation ein Ehegericht nach den materiellen Vorgaben einer auf den Lehren Luthers aufbauenden Eheordnung die Streitfälle entschied. ${ }^{26}$ In Kursachsen vergingen gut zwei Jahrzehnte, bevor auch dort das erste Ehegericht seine Arbeit aufnahm ${ }^{27}$ und bis zum Verlust der Kurwürde in der Jahrhundertmitte wurde auch keine materiell-rechtliche Normengrundlage geschaffen. Alles Gewicht wurde auf die Qualität des neuen Ehegerichts, des Wittenberger Konsistoriums gelegt. Mit hochkarätigen Gelehrten, zwei Professoren der juristischen und zwei Professoren der theologischen Fakultät wurde es besetzt und war damit in der Lage, auch ohne vorformulierte Normengrundlage, eine sowohl systematische als auch praxisgerechte Rechtsprechung auszuüben. Obwohl keine staatliche Maßnahme dem Wittenberger Konsistorium gegenüber der Konkurrenz einer Vielzahl anderer potentieller Entscheidungsträger für Ehesachen einen Vorrang gewährte, ${ }^{28}$ konnte es sich wegen der Qualität und Sachgerechtigkeit seiner Entscheidungen über alle politischen Wirren des Reformationsjahrhunderts hinweg und weit über die Landesgrenzen hinaus etablieren.

Die Rechtsprechung des Konsistoriums war von einem außerordentlich hohen Maß an Rationalität, Toleranz und die Orientierung an Individualinteressen geprägt. Diese Wertmaßstäbe treten als juristische Entscheidungsmaximen hervor und konkurrieren mit dem überlieferten christlichen Wertekanon. Besonders deutlich zeigt sich das in einem sehr typischen und kontrovers diskutierten Problembereich des frühen evangelischen Eherechts, bei den sog. ,heimliche(n) Verlöbnissen“, also allein von den Braut-

25 In unkomplizierten Fällen wurde eine Entscheidung der Pfarrer stillschweigend vorausgesetzt, in Zweifelsfällen sollten entweder die Amtleute, die kurfürstliche Kanzlei oder die Hofgerichte konsultiert werden. In der Praxis wandte man sich auch oft ohne weiteres an die Autoritäten der Reformation in Wittenberg, was bekanntlich Martin Luther über seine Arbeitsbelastung durch Eherechtsfälle klagen ließ. In einem Brief vom 2. November 1537 an den Pfarrer zu Prießnitz, Johann Wickmann, bat Luther, doch auch den "andern Pfarrherrn" zu sagen, sie mögen ihn von Ehesachen „verschonen“, denn er werde bereits "zuviel überschüttet", so daß er "schier kein Buch lesen noch schreiben" könne (WA, Briefwechsel, Bd. 8, Weimar 1938, S. 136, Nr. 3183). In ähnlicher Richtung in einem Brief an Leonhard Beyer vom 2. November 1535 (WA, Briefwechsel, Bd. 7, Weimar 1937, S. 322, Nr. 2270).

26 Überblick bei: Köhler, Walther, Die Anfänge des protestantischen Eherechts, ZRG KA 30 (1941), S. 271-310. Eingehend: Ders., Züricher Ehegericht und Genfer Konsistorium, Bd. 1, Das Züricher Ehegericht und seine Auswirkungen in der deutschen Schweiz zur Zeit Zwinglis, Leipzig 1932. Dazu: Stutz, Ulrich, Zu den Anfängen des evangelischen Eherechts, ZRG KA 22 (1933), S. 288-331. Und jetzt: Grünenfelder, Lukas, Das Zürcher Ehegericht. Eheschliessung, Ehescheidung und Ehetrennung nach der erneuerten Satzung von 1698, Zürich u. a. 2007.

27 Knapper Überblick bei: Frassek, Ralf, Liebe, Leid und Vernunft - Konstituierung und Praxis des frühen evangelischen Eherechts im Reformationsjahrhundert, in: ZRG KA 93 (2007), S. 372, 377 ff. Ausführliche Darstellung bei : Mejer, Otto, Anfänge des Wittenberger Consistoriums, in: ders., Zum Kirchenrechte des Reformationsjahrhunderts, Drei Abhandlungen, Hannover 1891.

28 Eine Arbeitsgrundlage des Wittenberger Konsistoriums wurde mit der Wittenberger Konsistorialordnung zwar nachträglich formuliert, jedoch formal niemals in Geltung gesetzt. Abdruck bei: Buchholtzer, Georg (Hrsg.), Constitution und Artickel des Geistlichen Consistorii zu Wittembergk... Anno MCXLII, Franckfurt an der Oder, Anno 1563. 
leuten - ohne Einverständnis ihrer Eltern - abgegebenen Eheversprechen. Die durch das kanonische Recht eröffnete Möglichkeit, eine wirksame Eheschließung allein durch den Konsens von Mann und Frau herbeizuführen, ${ }^{29}$ war am Vorabend der Reformation in das Zentrum der Kritik gerückt. Martin Luther vertrat die Ansicht, dass die heimlichen Verlöbnisse „nichts“ sein sollten, ihnen also keinerlei Wirksamkeit zugemessen werden sollte. Das Eherecht im Nachbarland Kursachsens, dem albertinischen Herzogtum Sachsen folgte strikt Luthers Ansicht. Schon bald nachdem im Herzogtum die Reformation eingeführt worden war, wurden hier mit den sog. Cellischen Ordnungen ${ }^{30}$ ausführliche und umfassende Normvorgaben formuliert, die heimliche Verlöbnisse für „unkreftig“, also nichtig erklärten. ${ }^{31}$ Das albertinische Eherecht sah sich mit dieser Praxis dem Folgeproblem gegenübergestellt, ob eine elterliche Zustimmungsverweigerung auch dann zu beachten sei, wenn diese ohne jegliche Begründung erfolgte. Dem Gedanken des Vierten Gebots folgend unterwarf man die Kinder ohne wenn und aber der Entscheidung ihrer Eltern. Die Möglichkeit, die Entscheidung der Eltern anhand rationaler Kriterien zu überprüfen, wurde nicht verfolgt. Der Begriff der elterlichen Gewalt und das christliche Gebot wurden - auch hier Luther folgend - im buchstäblichen Sinne interpretiert, eine Abwägung der Individualinteressen von Eltern und Kindern war also nicht vorgesehen. ${ }^{32}$

Ganz anders verfuhr man im ernestinischen Kurfürstentum, dem eigentlichen Wirkungsbereich Luthers, in dem man sich jedoch weit weniger an dessen Vorgaben hielt. Dass auch hier heimlichen Verlöbnissen entgegengewirkt werden sollte, kam zwar in einem Bestrafungsgebot deutlich zum Ausdruck, jedoch hütete man sich davor, solchen Verbindungen dogmatisch jegliche Wirksamkeit abzusprechen. Je nach den Umständen des Einzelfalles behielt man sich vor, zugunsten des Rechtsfriedens auch solche Verbindungen zuzulassen und die Betroffenen allenfalls durch kurze Arreststrafen für ihre Eigenmächtigkeit zu maßregeln. Auch Luther selbst sah sich gezwungen, konkrete Ein-

29 Frassek, Ralf, Consensus facit nuptias, in: Cordes, Albrecht, u. a. (Hrsg.), Handwörterbuch zur deutschen Rechtsgeschichte (HRG), Bd. 1, 2. Aufl., Sp. 884 f.

30 Benannt nach dem berühmten Kloster Altenzelle bei Nossen, wo diese Ordnung auf Anregung der Herzöge Moritz und August von Sachsen ab Dezember 1544 beraten und formuliert wurde. Ausführlich zur Entstehung G. Schleusner, Zu den Anfängen protestantischen Eherechts im 16. Jahrhundert. Mitteilung aus gleichzeitigen Akten, Zeitschrift für Kirchengeschichte Bd. 6 (1884), S. 397 ff.; Sehling, Emil, Die Evangelischen Kirchenordnungen des XVI. Jahrhunderts, Bd. 1, Sachsen und Thüringen, nebst angrenzenden Gebieten, S. 97 ff., Abdruck daselbst, S. 291 ff. Die Cellischen Ordnungen setzen sich aus drei Teilen, der Konsistorialordnung, dem Ehebedenken und der Kirchenordnung (im eigentlichen Sinne), zusammen.

31 "Nachdeme sichs ofte zutregt, wan die eltern ihre kinder fleissig auferzogen und sie, vor sich selbst, zu gelegner zeit zuverehelichen willig sein, das sich darzwischen ein junges dem andern aus unvorstande, trunkenheit, kopelei, ader in andere wege, wie das zukommen mag, zum ehestande vorpflicht, ahne vorbewust und bewilligung ihrer eltern, und aber got geboten hat, du solt vater und mutter ehren und gehorsam sein, welcher gehorsam in der heiligen geschrift, und kaiserlichen rechten under andern auch auf das eheliche vorpflichten gedeutet wirdet, so soll sölliche vorpflichtung, die ahne begrussung und bewilligung der eltern vorgenommen, in beiden consistoriis nach götlicher satzung, kaiserlicher ordnung und erforderung burgerlicher erbarkeit unkreftig und unkundig erkent werden" Sehling, Kirchenordnungen, Bd. 1, S. 292.

32 "Und sölchs sal ane unterscheid gehalten werden, ob auch gleich die eltern kaine ursache ihrer nicht bewilligung vorzuwenden hetten" Sehling, Kirchenordnungen, Bd. 1, S. 292. 
zelfallentscheidungen entgegen seiner ureigenen Ansicht zu treffen. ${ }^{33}$ Offensichtlich angeregt durch die Erfahrungen der Eherechtspraxis wurde den Eltern der potentiellen Brautleute auferlegt, tragfähige Gründe für die Verweigerung einer Zustimmung vorzubringen, um so Willkürentscheidungen und dadurch möglicherweise provozierten Rechtsverstößen vorzubeugen. ${ }^{34}$ Die ,elterliche Gewalt“ sollte nur dann Wirkung entfalten können, wenn sie auch pflichtgemäß ausgeübt wurde. Das christliche Gebot, die Eltern zu ehren, wurde hier also mit einer korrespondierenden Verpflichtung in der Gegenrichtung verknüpft, um eine gerechte Einzelfallentscheidung zu gewährleisten. Wurden keine tragfähigen Gründe vorgebracht, behielt sich das Konsistorium selbst die Entscheidung vor, das Elterneinverständnis zu ersetzen. Bemerkenswert ist vor allem die Begründung für diese Vorgehensweise: „Ohne genügsame Ursache sollen die Eltern die Kinder, die sich einander ehelich versprochen hatten, nicht daran hindern, Liebe und Leid miteinander zu teilen“. ${ }^{35}$ An anderer Stelle lautet die Formulierung sogar „Lust und Liebe zusammen zu haben". 36

Das Konsistorium traf diese Entscheidung zugunsten der Kinder im vollen Bewusstsein, dass ,in der heiligen göttlichen Schrift den Eltern die Ehre gegeben ist, dass die Kinder sich ohne ihr Wissen und ihren Konsens nicht verloben sollen. "37 Sehr deutlich kommt in den Formulierungen die Zielsetzung zum Ausdruck, das individuelle Glück der Betroffenen mit den traditionellen christlichen Wertvorstellungen in sachgerechten Einklang zu bringen. Die Brautleute ohne ausreichenden Grund daran hindern zu wollen, „Liebe und Leid“ mit dem selbst gewählten Lebenspartner teilen zu dürfen, wurde trotz des Vierten Gebotes als „Tyrannei“ bewertet. Ausdrücklich als nicht für eine Verweigerung ausreichender Grund wurde der Fall genannt, ,dass Eins dem Andern vielleicht nicht reich genug sei“ 38

33 Zum Umgang Luthers mit der Tatsache sich mit dieser Ansicht rechtspraktisch nicht durchsetzen zu können: Frassek, Eherecht und Ehegerichtsbarkeit, S. $52 \mathrm{ff}$.

34 "Nach dem aber auch die eltern ires gewalts zu zeiten missbrauchen, do zwei von gleichem alter, auch von leben unbescholten, gesundes leibs, und sonst von stande und herkommen einander nicht ungemes, lust und liebe zusammen haben, nemen ihnen die eltern ursach, das eins dem andern nicht reich gnug, oder sehen zu weilen mehr ihr eigen, denn der kinder nutz an, und wollen die kinder an irer verhairatung hindern, welchs denn mehr ein tyrannei, denn offentlicher gewalt zu achten, so erfolgt auch daraus allerlei unrath, wie in teglicher Erfahrung befunden" Sehling, Kirchenordnungen, Bd. 1, S. 209.

35 "Den obwol in der heiligen göttlichen schrifft den Eltern die ehere gegeben ist, das die kinder ohne Jre verweissen vnndt Consens sich nicht vorloben sollen. So ist doch gleichwol dieselbige zuuerstehen, das die Eltern ohne gnugsame vrsach die kinder so sich ehelich vorsprochen haben, Liebe vnndt Lidt zusammen haben nicht hindern sollen, hette sie aber gnugsame vrsach warumb sie darin nicht verwilligen wolte, das sie auch dieselbigen anzeigen solte, vndt so ihr aus den hernachmalß neben andern was von beiden teilen eingebracht zuschicket, so wolt wir in der hauptsachen erkennen was recht ist." Lutherhaus Wittenberg, Wittenberger Consistorial-Akte in Ehesachen, Katalog S. 165, 1371-1372 ("Wittenbergisches Buch", auch als „Codex Schleusner" bezeichnet), Fall Nr. 5, Bl. 66v f. In der Sachlage und der ausgesprochenen Rechtsfolge vergleichbar auch Fall Nr. 21, Bl. 72 rf. - Zum Quellentext: Frassek, Ralf, Das "Wittenbergische Buch" - Ein bedeutender Quellentext für das frühe evangelische Eherecht, in: Neues Archiv für Sächsische Geschichte, Bd. 74/75 (2003/2004), S. 67-97.

36 Sehling, Kirchenordnungen, Bd. 1, S. 209.

37 Wittenbergisches Buch, Fall Nr. 5, Bl. 66 vf.

38 Sehling, Kirchenordnungen, Bd. 1, S. 209. 
Als beachtenswerter Grund für eine Verweigerung anerkannt wurde dagegen ein als unnatürlich empfundener Altersunterschied der Verlobten wie im Fall eines Achtzehnjährigen, dessen Vater die Zustimmung zur Heirat mit einer fünfzigjährigen Witwe verweigert hatte. ${ }^{39}$ Vorrangig angestrebt wurde jedoch, den Ausspruch der Unwirksamkeit $\mathrm{zu}$ vermeiden und stattdessen auf eine Konsenslösung unter den Beteiligten hinzuwirken. So ermahnte das Konsistorium den Vater einer Braut, „Schimpf und Schande“ zu bedenken, die der Tochter drohten, wenn seine Verweigerung das letzte Wort bliebe. ${ }^{40}$

Aus dem aufgezeigten sensiblen und gleichzeitig an die gesellschaftlichen Verhältnisse der Zeit angepassten Umgang mit religiös intendierten Normvorgaben können wertvolle Erkenntnisse für den Diskurs über aktuelle Fragen unserer multikulturellen Gesellschaft, konkret im Spannungsfeld zwischen Christentum und Islam, gewonnen werden. Als hervorstechendes Beispiel sei hier nur das Problem der Zwangsverheiratung und arrangierter Ehen genannt. ${ }^{41}$ Die Praxis der frühen evangelischen Ehegerichtsbarkeit verdeutlicht, wie stark eine Rechtsmaterie nicht von der Form und dem Umfang der konstituierten Normen, sondern durch die Erfahrungswelt und Integrität der berufenen Entscheidungsträger geprägt wird. Selbst wohlbedachte und eindeutige Entscheidungsprämissen können die unabsehbar vielfältigen Herausforderungen des menschlichen Alltags nur dann bewältigen, wenn sie mit dem notwendigen kritischen Sachverstand eingesetzt werden.

\section{Schlusswort}

Ohne eine reflektive Kontrolle der aktuellen Rechtsentwicklung besteht die Gefahr, dass kritisches Wissen verloren geht und lediglich die Reproduktion von Vorhandenem vorherrscht. Recht, insbesondere Privatrecht ist jedoch etwas Lebendiges, das vernunftorientierter und sachgerechter Innovationen bedarf. Es muss sich stets an die sich verändernden Lebensumstände anpassen können. Und dazu bedarf es Vielfalt, nicht Einfalt der Meinungen, aus denen für die Gestaltung der Zukunft geschöpft werden kann.

Für die juristischen Fakultäten stellt sich die Herausforderung, die Rechtsgeschichte ebenso wie die anderen Grundlagenfächer nicht als etwas Abgegrenztes, in den ersten

39 „Berichten vnndt bekennen darauf dieweil d. Rathsmeister Jobst geleucklin anzeigt, d. sein Sohn vber 18 Jahren nicht alt, die widtwe 50 Jhar. Vf Jr haben solte, das sie dan beyderseits nicht vormeinen, darauß d. vater sorge treget, das die vngleicheitt zu seines Sohnes fall vnndt sünden ein vrsach werden möchte, neben dem das auch andere bewegliche vrsachen durch Jhnen vorgewandt warumb er ihm d. ehegelübd nicht willigen wolle, So thutt er sich in dem seines veterlichen gewalts vnndt rechten zugebrauchen, deme auch der Sohn zu gehorchen von dem vater seine gebürliche ehre vnndt gehorsam zuleisten schuldigk" Wittenbergisches Buch, Fall Nr. 66, Bl. 95 v.

$40, \ldots$ das er in das ehegelübde seiner tochter nicht gewilliget noch aus erheblichen vrsachen drein willigen möchte oder wolte. So würden solche sponsalia so ohne sein consens vnd bewilligung geschehen, wo man in nicht dazu vermögen möchte, das man doch aus christlicher liebe nochmals mit vleis versuchen sol, mit erinnerung was vor gefahr vnd schimpf seiner tochter gleichwol darauff stünde." Wittenbergisches Buch, Bl. $58 \mathrm{v}$.

41 Berührungs- und Anknüpfungspunkte zeigen sich hier zu der im Rahmen des Münsteraner Exzellenzclusters "Religion und Politik" betriebenen Forschung, insbesondere dessen Forschungsfeldern "A - Normativität" und "C - Integrative Verfahren". 
Studiensemestern Abzuschichtendes zu begreifen, sondern sie als verbindendes Glied anzuerkennen, das seinen Wert in allen Bereichen des geltenden Rechts besitzt. Es ist und bleibt eine der wichtigsten Aufgaben der Universität, bei den Studierenden und vielleicht auch bei so manchem Lehrenden der Furcht vor der Freiheit, kritisch zu denken, mit allen Kräften entgegenzuwirken. 